\title{
Does genetic heterogeneity account for the divergent risk of type 2 diabetes in South Asian and white European populations?
}

\author{
Zahra N. Sohani • Wei Q. Deng • Guillaume Pare • \\ David Meyre • Hertzel C. Gerstein • Sonia S. Anand
}

Received: 19 May 2014 / Accepted: 22 July 2014 / Published online: 22 August 2014

(C) The Author(s) 2014. This article is published with open access at Springerlink.com

\begin{abstract}
Aims/hypothesis South Asians are up to four times more likely to develop type 2 diabetes than white Europeans. It is postulated that the higher prevalence results from greater genetic risk. To evaluate this hypothesis, we: (1) systematically reviewed the literature for single nucleotide polymorphisms (SNPs) predisposing to type 2 diabetes in South Asians; (2) compared risk estimates, risk alleles and risk allele frequencies of predisposing SNPs between South Asians and white Europeans; and (3) tested the association of novel SNPs discovered from South Asians in white Europeans.

Methods MEDLINE, Embase, the Cumulative Index to Nursing and Allied Health Literature (CINAHL) and the Cochrane
\end{abstract}

Electronic supplementary material The online version of this article (doi:10.1007/s00125-014-3354-1) contains peer-reviewed but unedited supplementary material, which is available to authorised users.

Z. N. Sohani · W. Q. Deng • G. Pare • D. Meyre • H. C. Gerstein •

S. S. Anand $(\bowtie)$

Population Genomics Program, Department of Clinical

Epidemiology and Biostatistics, McMaster University,

1280 Main St W, Hamilton, ON, Canada L8S 4L8

e-mail: anands@mcmaster.ca

Z. N. Sohani - W. Q. Deng • G. Pare • D. Meyre · S. S. Anand Chanchlani Research Centre, McMaster University,

Hamilton, ON, Canada

Z. N. Sohani $\cdot$ W. Q. Deng • G. Pare • D. Meyre $\cdot$ H. C. Gerstein •

S. S. Anand

Population Health Research Institute, Hamilton Health Sciences,

Hamilton, ON, Canada

G. Pare $\cdot$ D. Meyre

Department of Pathology and Molecular Medicine, McMaster

University, Hamilton, ON, Canada

H. C. Gerstein · S. S. Anand

Department of Medicine, McMaster University,

Hamilton, ON, Canada registry were searched for studies of genetic variants associated with type 2 diabetes in South Asians. Meta-analysis estimates for common and novel bi-allelic SNPs in South Asians were compared with white Europeans from the DIAbetes Genetics Replication And Meta-analysis (DIAGRAM) consortium. The population burden from predisposing SNPs was assessed using a genotype score.

Results Twenty-four SNPs from 21 loci were associated with type 2 diabetes in South Asians after meta-analysis. The majority of SNPs increase odds of the disorder by $15-35 \%$ per risk allele. No substantial differences appear to exist in risk estimates between South Asians and white Europeans from SNPs common to both groups, and the population burden also does not differ. Eight of the 24 are novel SNPs discovered from South Asian genome-wide association studies, some of which show nominal associations with type 2 diabetes in white Europeans.

Conclusions/interpretation Based on current literature there is no strong evidence to indicate that South Asians possess a greater genetic risk of type 2 diabetes than white Europeans.

Keywords Epidemiology · Ethnicity · Genetic risk · Meta-analysis · South Asian · Type 2 diabetes · White Europeans
Abbreviations
DIAGRAM DIAbetes Genetics Replication And
Meta-analysis
GWAS Genome-wide association studies
HWE Hardy-Weinberg equilibrium
LD Linkage disequilibrium
RAF Risk allele frequency
SNP Single nucleotide polymorphism 


\section{Introduction}

Type 2 diabetes is a metabolic disorder resulting from the interplay between insulin secretion and action. The prevalence of type 2 diabetes is increasing globally [1]. Currently, there are 61 million people with diabetes in the Indian subcontinent, a number that will rise to 100 million by the year 2030 , representing $25 \%$ of the world's burden [2, 3]. South Asians develop metabolic abnormalities, such as hyperglycaemia, low HDL-cholesterol, and elevated triacylglycerol, at a lower BMI and younger age than white Europeans [4-6]. It is hypothesised that the higher prevalence of type 2 diabetes results from a greater genetic predisposition among South Asians [7, 8], either because of larger risk estimates for each single nucleotide polymorphism (SNP) or increased frequency of risk alleles. This hypothesis has not been systematically evaluated.

The purpose of this systematic review is to: (1) establish risk estimates for SNPs predisposing South Asians to type 2 diabetes; (2) compare risk estimates, risk alleles and risk allele frequencies (RAFs) of type 2 diabetes predisposing SNPs between South Asians and white Europeans; and (3) explore the association of novel SNPs discovered from South Asians in a large cohort of white Europeans.

\section{Methods}

Systematic review of studies assessing genetic risk of type 2 diabetes in South Asians

Search strategy and selection criteria MEDLINE, Embase, the Cumulative Index to Nursing and Allied Health Literature (CINAHL) and the Cochrane registry (from inception to 17 June 2013; MEDLINE and Embase searched using OvidSP) were searched for studies of genetic variants associated with type 2 diabetes in South Asians. South Asians were defined as individuals originating from India, Pakistan, Bangladesh or Sri Lanka. The search strategy was developed in consultation with a research librarian and did not restrict by type of genetic variant, language of study or study design (i.e. case-control, cohort, cross-sectional). The full search strategy is presented in electronic supplementary material (ESM) Table 1. Experts were consulted, and reference lists of included articles as well as relevant excluded articles were searched. Two reviewers (ZNS and WQD) independently assessed each study for eligibility based on four questions: (1) is at least one study population South Asian; (2) is type 2 diabetes the outcome studied; (3) is the exposure a genetic variant; and (4) is this a genetic association study? Disagreements were independently resolved by a third reviewer (SSA). Articles that passed the screening phase were reviewed in depth.

Full-text review and data extraction Conference abstracts, narrative reviews and other systematic reviews were excluded after full-text review. Primary studies investigating genetic variants other than bi-allelic SNPs (e.g. insertions, deletions, length polymorphisms, haplotypes and complex tri-allelic SNPs) or with fewer than 25 cases were excluded. These restrictions were implemented to ensure that included studies could be combined and were of sound quality. Additionally, to ensure robustness of our results, SNPs from included studies carried forward to meta-analysis were limited to those that previously reached genome-wide significance in any ethnicity. If datasets were published more than once, publication with the largest sample size or the one recommended by the senior author was used for meta-analysis. Two reviewers conducted a full-text assessment and extracted data on participant characteristics (sample size, age, BMI, fasting glucose, waist and hip circumferences and \% men), study design (study methods, region) and results (SNP, risk allele, RAF in cases and controls, and risk estimate with a $95 \% \mathrm{CI}$ ). For genome-wide association studies (GWAS) in South Asians, SNPs associated with type 2 diabetes at $p<10^{-3}$ were considered for meta-analysis.

Quality assessment of included studies Using recommendations from the STrengthening the REporting of Genetic Association Studies (STREGA) guidelines [9], the following three components were considered for quality assessment: testing for Hardy-Weinberg equilibrium (HWE) in controls [10], reporting of genotyping call rate or appraisal of genotyping quality by duplication, and sources of case and control ascertainment.

Statistical analysis Agreement between reviewers was reported with Cohen's $\kappa$ statistic. Allelic ORs were calculated using RAFs and sample size for cases and controls and assessed for significance using Fisher's exact test. The ORs were not adjusted for any covariates. ORs for SNPs in linkage disequilibrium (LD) $\left(r^{2}>0.8\right)$ obtained from independent cohorts were meta-analysed using a random-effects model weighted by inverse variance. LD was estimated using 1000 Genome Centre d'Etude du Polymorphisme (CEU) (Utah residents with northern and western European ancestry) data from SNAP (Broad Institute, Cambridge, MA, USA) [11]. A twosided $\alpha$ level of 0.05 was considered significant. For a list of SNPs in LD, see ESM Table 2. Summary RAFs are presented as averages, weighted by sample size, of all included reports. Heterogeneity was estimated using $I^{2}$ statistic, which indicates the proportion of total variation in estimates attributed to heterogeneity, as well as the $Q$ statistic [12]. A cut-off of $25 \%$ for $I^{2}$ was used to represent minimal heterogeneity, $50 \%$ to represent moderate and $75 \%$ to represent high heterogeneity. Quanto, Los Angeles, CA, USA (version 1.2.4) was used for sample size and power calculations assuming a disease prevalence of $10 \%$ and an additive model of inheritance. All other statistical analyses were conducted in $\mathrm{R}$ (version 3.0.2). 
Comparison of meta-analysed SNPs in South Asians with white Europeans

Estimates for SNPs associated with type 2 diabetes in this meta-analysis were retrieved for white Europeans from the DIAbetes Genetics Replication And Meta-analysis (DIAGRAM) consortium. Specific details on the consortium have been previously described [13]. Briefly, DIAGRAM stage 1 is a publicly available database of 12 GWAS with 12,171 cases and 56,862 controls. DIAGRAM authors tested SNPs with minor allele frequency $>1 \%$ for association with type 2 diabetes under an additive model. The authors combined summary estimates from the 12 GWAS using fixedeffect inverse-variance-weighted meta-analysis.

Effect sizes from white Europeans in DIAGRAM and South Asians from this meta-analysis were transformed to natural logs and compared using a $Z$ test. RAFs in white Europeans were acquired from published GWAS (www. genome.gov/gwastudies/). Additionally, a genotype score of SNPs present in both groups was constructed to compare population burden from these SNPs. The genotype score was constructed as below:

Genotype score $=\Sigma\left[\log _{e}\left(\mathrm{OR}_{\mathrm{i}}\right) \times \mathrm{RAF}_{\mathrm{i}}\right]$

Variance for the genotype score was estimated as:

Variance $=\Sigma\left\{\mathrm{RAF}_{\mathrm{i}}^{2} \times\right.$ variance $\left.\left[\log _{e}\left(\mathrm{OR}_{\mathrm{i}}\right)\right]\right\}$

Genotype scores from both ethnicities were compared using a $Z$ test.

Testing novel SNPs discovered from South Asians GWAS in white Europeans

Four GWAS have been conducted in South Asians [14-17], which collectively identified nine SNPs $\left(p<5 \times 10^{-8}\right)$. All studies used a case-control design. Associations with type 2 diabetes for eight of the nine SNPs have not been independently discovered from GWAS in any other ethnicity. We compared the association for these eight novel SNPs with type 2 diabetes in white Europeans from DIAGRAM. An overview of the study design is presented in Fig. 1a.

\section{Results}

Systematic review and meta-analysis

Search yields A total of 3,898 articles were originally screened, of which 170 were carried to full-text review. Ninety-four studies met the inclusion and exclusion criteria, and 38 studies comprising 31 independent cohorts contained data on SNPs with evidence of genome-wide significance in any ethnicity. These 38 studies were included in the systematic review. Figure $1 \mathrm{~b}$ depicts the selection process and lists reasons for exclusion at each stage. There was excellent agreement on study inclusion between reviewers (unweighted $\kappa=0.898)$.

Study characteristics All included studies were in English and published in 2004 or later. Most large datasets $(>2,500$ people) were published after 2008. Eight of the 31 cohorts were from North India, four from South India, five from Pakistan, one from the Eastern region (Orissa), four from Indians residing in Singapore, two from Sri Lanka, one from Indians residing in Trinidad, one from Indians residing in Mauritius and one from all over the South Asian subcontinent; four were unspecified.

Participant characteristics Studies eligible for analysis included 29,618 cases and 40,329 controls. Controls were described as healthy individuals who were normoglycaemic and/ or non-diabetic. Men accounted for $54 \%$ of cases and $53 \%$ of controls. Age ranged from 34 to 62 years in cases [18, 19], and from 28 to 62 years in controls $[18,20]$. BMI was reported for cases and controls in $79 \%$ of studies and ranged from 25.0 to $31.9 \mathrm{~kg} / \mathrm{m}^{2}$ in cases [21, 22] and from 19.4 to $28.1 \mathrm{~kg} / \mathrm{m}^{2}$ [23, $24]$ in controls. A majority of studies reported fasting glucose: $58 \%$ in cases (ranging from 6.41 to $10.60 \mathrm{mmol} / \mathrm{l}$ ) $[25,26]$ and $63 \%$ in controls (ranging from 4.60 to $6.89 \mathrm{mmol} / \mathrm{l}$ ) [14, 27]. Very few datasets reported additional clinical information; $29 \%$ reported waist circumference in cases and controls. Only three studies reported hip circumference. ESM Table 3 provides a summary of all included studies.

Quality assessment Agreement with HWE in controls, genotyping call rate and source of controls and cases for included studies are summarised in ESM Table 4. One study did not report compliance with HWE [28]. SNPs in one study deviated from HWE [29]; rs2237892 and rs2237897 deviated from HWE at $p=0.002$ and $p=0.001$, respectively. The SNPs were excluded from analysis because the HWE threshold in the primary report was fairly conservative. Six studies did not appraise genotyping quality [28, 30-34]. Furthermore, three studies had a genotyping call rate below $95 \%$ for all reported SNPs [29, 35, 36].

Risk estimates for SNPS associated with type 2 diabetes in South Asians Thirty SNPs with previous evidence of genomewide significance in other ethnicities were available for metaanalysis. Pooled estimates were significant for 15 of the 30 SNPs (Fig. 2). Summary ORs, on the whole, suggested between a 1.15- and a 1.35-fold increase in susceptibility for type 2 diabetes per risk allele. Surprisingly, the risk alleles for both $K C N Q 1$ polymorphisms substantially increased the odds 
Fig. 1 (a) Overview of the study design. (b) Flow diagram of the systematic review of South Asian literature investigating genetic variants predisposing to type 2 diabetes (first objective of study). T2DM, type 2 diabetes mellitus a

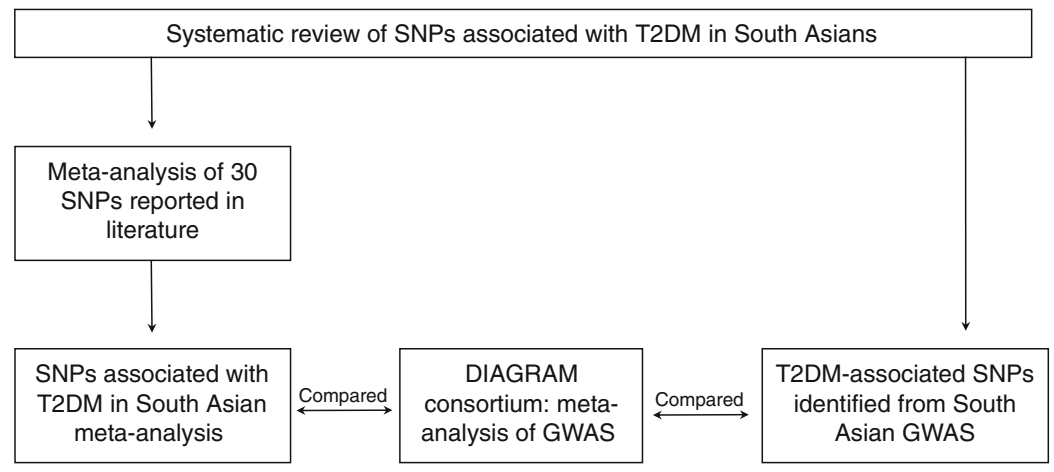

b
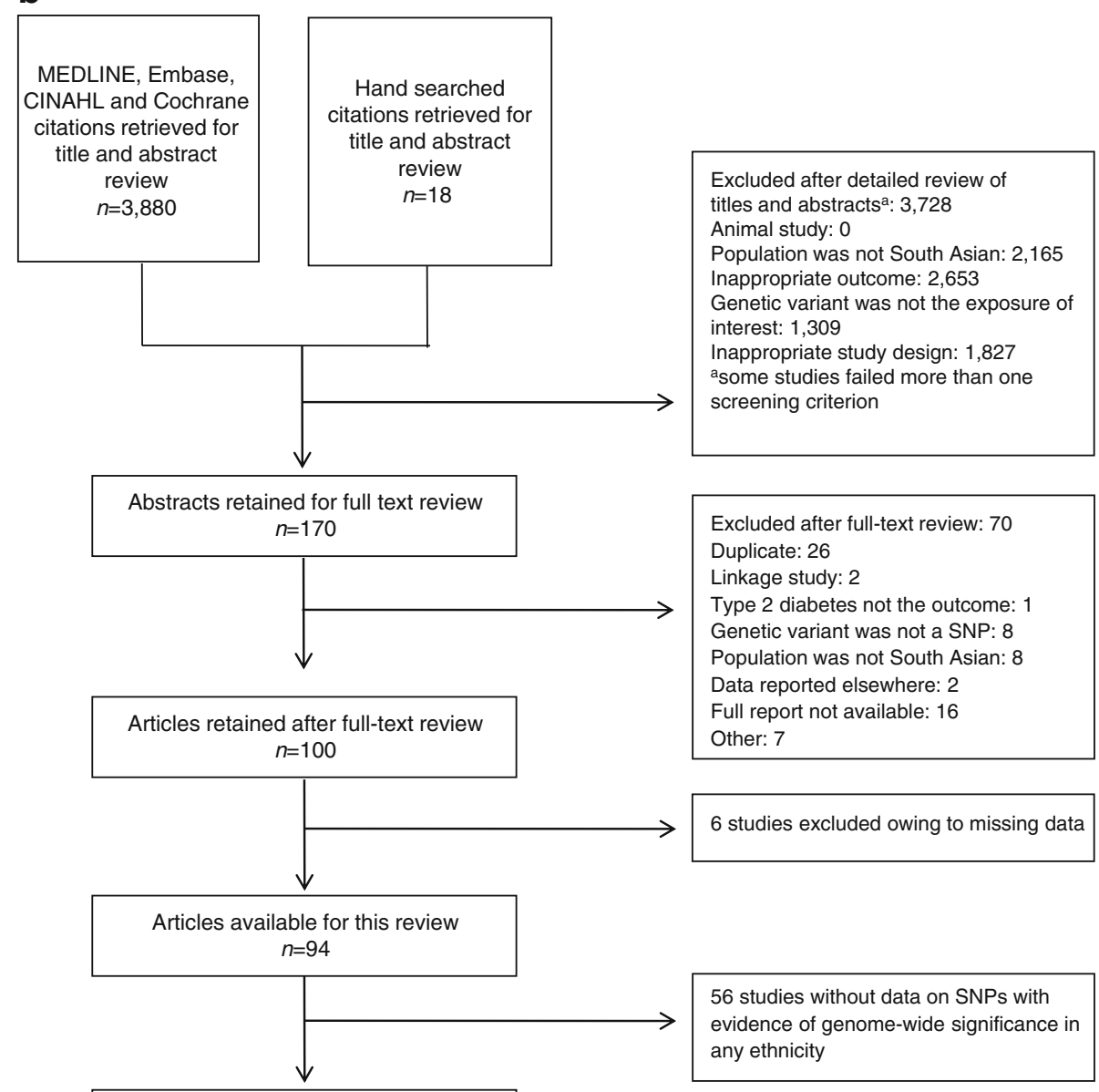

Articles included in this review $n=38$ of type 2 diabetes (rs2237892 OR 1.62, 95\% CI 1.01-2.59; rs2237897 OR 2.19, 95\% CI 1.25-3.82). High degrees of between-study heterogeneity were evident for CDKAL1 rs7754840 $\left(I^{2}=70 \%, Q=19.99, p<0.01\right)$ and $T C F 7 L 2$ rs7903146 $\left(I^{2}=90 \%, Q=99.52, p<0.01\right)$. In the TCF7L2 meta-analysis, there was some evidence that larger studies had more conservative estimates, although Chauhan et al [35] and Uma Jyothi et al [25] were exceptions. The smallest study $(n=$
40 case/control pairs) depicted an association that was directionally inconsistent with the others [22]. Heterogeneity estimates did not change much without this outlier $\left(I^{2}=90 \%, Q=90.31, p<0.01\right)$. Additional sources of heterogeneity could include consanguinity [34, 37]. One very obvious outlier existed in the meta-analysis for CDKAL1 (OR 2.22, 95\% CI 1.64-2.98) [37]. Heterogeneity estimates without this outlier diminished 
substantially $\left(I^{2}=0 \%, Q=2.80, p=0.73\right)$. None of the outliers was excluded from our final analysis.

A majority of genes associated with type 2 diabetes from this meta-analysis (ADCY5, CDKAL1, CDKN2A/B, HHEX, $I G F 2 B P 2, S L C 30 A 8, T C F 7 L 2$ and KCNQ1) are involved in pancreatic beta cell function, while two are implicated in insulin sensitivity $(P P A R G)$ and adiposity $(F T O)$. This is not surprising as insulin secretion is a more heritable trait than insulin action [38]. In addition to the above, eight novel SNPs were identified from South-Asian-only GWAS; these are discussed later in this paper.

Comparison of effect sizes, RAF, and risk alleles between South Asians and white Europeans

Meta-analysis estimates in South Asians were compared with white Europeans from DIAGRAM; no significant differences were identified for most SNPs $(p<0.05)$ (Fig. 2), although two SNPs showed heterogeneity (KCNQ1 rs2237897 and IGF2BP2 rs4402960). South Asians had a slightly smaller OR for IGF2BP2 rs4402960 (South Asians: 1.07, 95\% CI 1.04-1.09; white Europeans: 1.13, 95\% CI 1.09-1.17).
Figure 3 presents a Venn diagram of overlapping type 2 diabetes predisposing genes in South Asians and white Europeans, as well as genes unique to both groups.

Variation in RAFs between South Asians and white Europeans was observed, but no consistent trend was evident; South Asians did not consistently have an RAF greater than white Europeans (Fig. 2). While the risk allele for HHEX was the same in both ethnicities, the RAF differed; the risk allele is the minor allele in South Asians, but the major/common allele in white Europeans. Risk alleles for six SNPs (SLC30A8 rs13266634, ADCY5 rs11708067, PPARG rs1801282, CHCHD9 (also known as CHCHD2P9) rs13292136, KCNQ1 rs2237897 and $C D K N 2 A / B$ rs10811661) were major/common alleles in both ethnicities.

Testing novel SNPs discovered from South Asian GWAS in white Europeans

The systematic review included four GWAS in South Asians, which identified nine SNPs associated with type 2 diabetes at genome-wide significance. With the exception of $H M G 20 \mathrm{~A}$ rs7178572 (South Asian OR: 1.09, 95\% CI 1.06-1.12; white

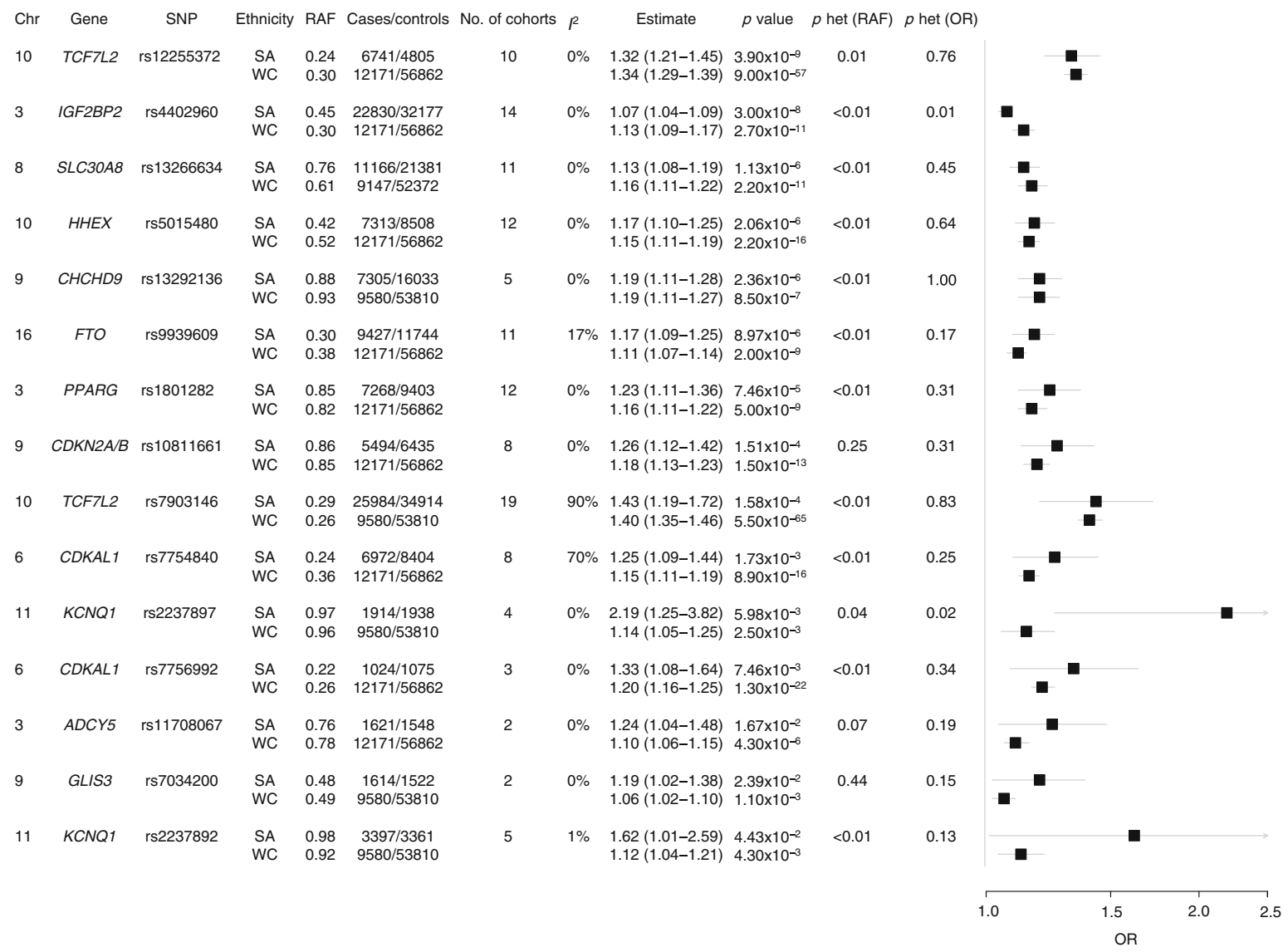

Fig. 2 Forest plot of SNPs associated with type 2 diabetes in South Asians and comparison with white Europeans from DIAGRAM. Chr, chromosome; het, heterogeneity; no., number; SA, South Asian; WC, white European 
Fig. 3 Venn diagram of SNPs common to South Asians and white Europeans and SNPs unique to both groups. CENTD2 is also known as $A R A P 1$. The green box includes genes with GWAS evidence for association with type 2 diabetes; the pink box includes genes with GWAS evidence in white Europeans and association with type 2 diabetes in this meta-analysis; and yellow with genes identified from a transethnic meta-analysis of South Asians and white Europeans [49]

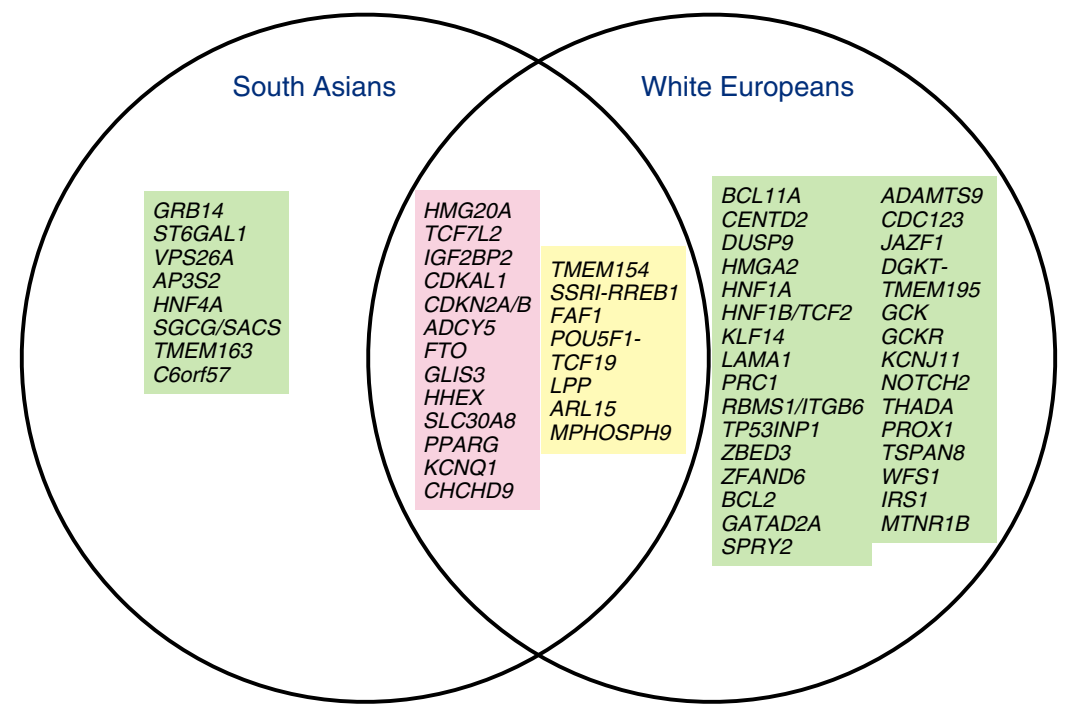

European OR: 1.08, 95\% CI 1.05-1.10) [14, 39], the remaining eight SNPs have not been discovered in any other ethnic group. Five of the eight (GRB14 rs3923113, VPS26A rs1082295, $H N F 4 \alpha$ [also known as HNF4A] rs4812829, ST6GAL1 rs16861329, and AP3S2 rs2028299) were independently replicated in South Asians [15].

We tested the eight novel SNPs for association with type 2 diabetes in DIAGRAM. SGCG rs9552911 was monomorphic in white Europeans. The remaining SNPs were directionally consistent with South Asian estimates and three (AP3S2 rs2028299, GRB14 rs3923113 and $H N F 4 \alpha$ rs4812829) were nominally $(p<0.05)$ associated with type 2 diabetes (Table 1$)$ in DIAGRAM. The other four were not associated with type 2 diabetes in white Europeans. No consistent trend in RAFs was observed. The risk allele for TMEM163 rs6723108 is the same in both ethnicities, but the RAF differs. Specifically, the risk allele is the minor allele in white Europeans, but the major/ common allele in South Asians.

\section{Population burden}

All sixteen SNPs, which were significantly associated with type 2 diabetes in South Asians, were included in the genotype score (ADCY5, CDKAL1 rs7754840 and rs7756992, CDKN2A/2B, FTO, GLIS3, HHEX, PPARG, SLC30A8, TCF7L2 rs7903146 and rs12255372, CHCHD9, KCNQ1 rs2237892 and rs2237897, IGF2BP2 and HMG20A SNPs from Table 1 and Fig. 2). RAFs for both ethnicities were weighed by white European summary ORs from DIAGRAM because our primary analysis showed that individual risk estimates did not vary, and due to a larger sample, we expect the white European estimates to be more precise. Using this approach, no difference in the population burden was observed ( $p=0.85$ ) (Fig. 4).

\section{Discussion}

Twenty-four SNPs were associated with type 2 diabetes in South Asians, eight of which were novel, discovered from GWAS. There were some variations in RAFs but the effect sizes for common SNPs did not differ between the ethnic groups. Interestingly, only three of the novel SNPs discovered from the South Asian GWAS were nominally associated with type 2 diabetes in white Europeans. Overall, the population burden from type 2 diabetes SNPs estimated using a genotype score appears to be comparable in both ethnicities.

SNPs associated with type 2 diabetes from meta-analysis of South Asian studies

Meta-analysis of risk alleles for most SNPs increased the odds of type 2 diabetes by $15-35 \%$ among South Asians. Notable exceptions were the $K C N Q 1 \mathrm{SNPs}$, which showed prominent ORs for type 2 diabetes. $K C N Q 1$ SNPs have been shown as a more significant contributor to type 2 diabetes than other loci in other Asian populations [40] and because both SNPs (in weak LD) with larger ORs are located on the same gene, it appears that $K C N Q 1$ may truly have a stronger signal in South Asians than in white Europeans. However, the exceptionally large effect size for $K C N Q 1$ rs2237897 is likely to be inaccurate, given the wide CIs and inconsistency with GWAS estimates from Europeans and East Asians (OR 1.33, 95\% CI 1.24-1.41) [41]. Moreover, the KCNQ1 rs2237897 association was reported in only two studies among South Asians and thus could be a product of the winner's curse [42].

Fifteen GWAS signals for type 2 diabetes in white Europeans, East Asians, and Singaporean Malay [43-46] were not associated with type 2 diabetes in our South Asian metaanalysis. This may reflect low power to detect similar effect 


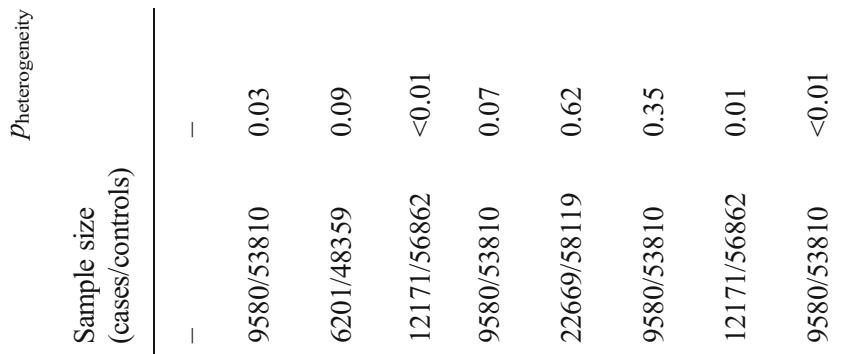

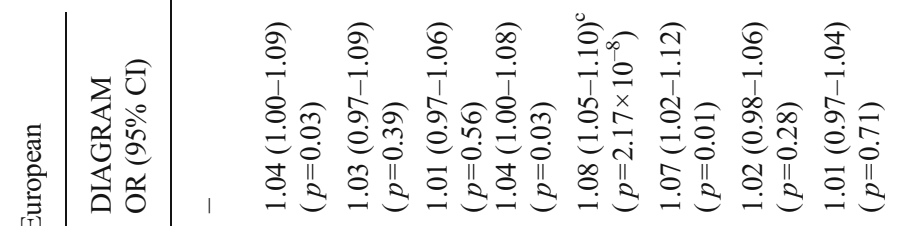
要

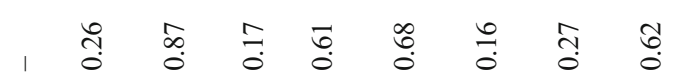

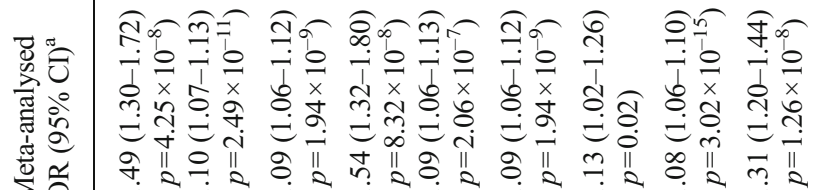

สิกสิ

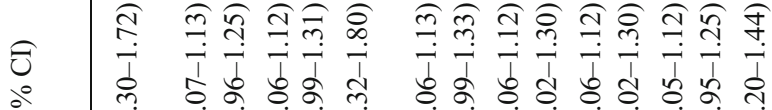

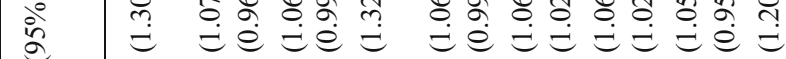

음 守

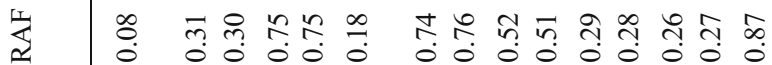

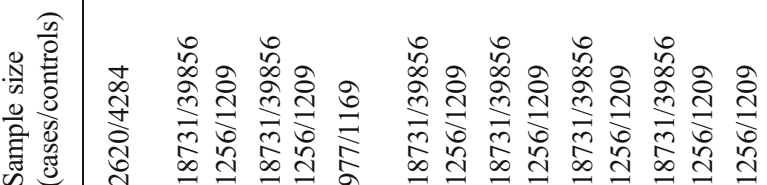

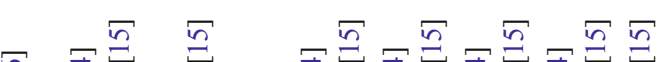

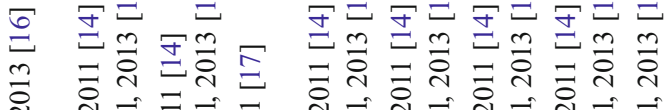

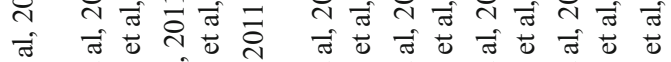

$\overrightarrow{0}$ एँ

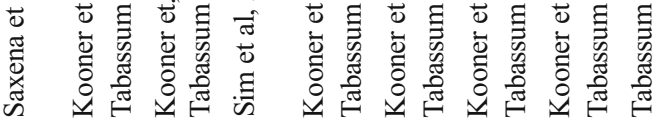

$\frac{\pi}{2}$

$\frac{\sqrt{n}}{2}$

玄 ๓

(1)

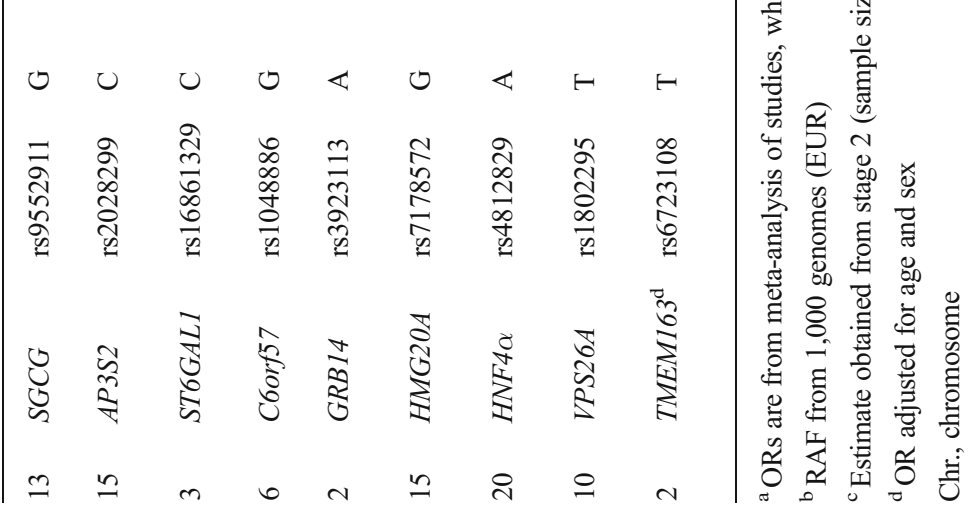




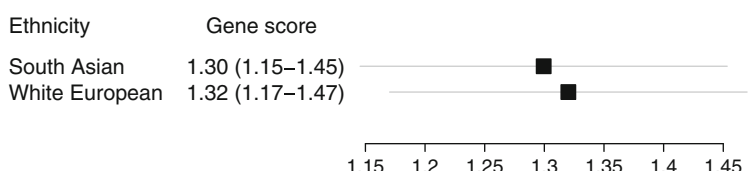

Fig. 4 Gene score of SNPs in South Asians and white Europeans. The gene score was constructed using effect estimates and RAFs from SNPs common to both groups

sizes for ADAMTS9 rs4607103, CDC123 rs12779790, JAZF1 rs864746, KCNQ1 rs231362, DGKT-TMEM195 rs2191349 (also known as $A G M O$ ), GCK rs1799884, GCKR rs780094, MTNR1B rs10830963, PROX1 rs340874, and TSPAN8 rs7961581 (Table 2) and either a true lack of association for KCNJ11 rs5219, KCNQ1 rs2237895, NOTCH2 rs10923931, THADA rs7578597 and WFS1 rs10010131 or significant between-study heterogeneity since we were adequately powered for the last five SNPs (Table 2). KCNJ11 rs5219 in particular was close to significance (OR 1.19. 95\% CI 0.98 $1.45)$ and demonstrated a high degree of heterogeneity $\left(I^{2}=81 \%, p<0.01\right)$.

Comparison of effect sizes, RAF and risk alleles between South Asians and white Europeans

In general when comparing effect sizes of SNPs associated with type 2 diabetes from our meta-analysis with DIAGRAM estimates in white Europeans, the risk from SNPs predisposing to type 2 diabetes did not differ substantially between the groups. However, the point estimates were more precise and CIs tighter among the white Europeans because of the larger sample size. Our observation of no significant difference in risk estimates is not surprising because SNPs evaluated in South Asians are selected for homogeneity as they were first discovered in white Europeans and then replicated in South Asians. If there is a difference in genetic risk between the ethnic groups, it probably does not result from polymorphisms common to both groups. The results of our paper are supported by those recently published by DIAGRAM, the Asian Genetic Epidemiology Network Type 2 Diabetes (AGENT2D) Consortium, and the South Asian Type 2 Diabetes (SAT2D) Consortium [49]. Their study also found effect estimates for common SNPs predisposing to type 2 diabetes to be homogenous among South Asians and white Europeans. In addition to the recently published study, we show that the gene score, which measures population burden in both groups, does not differ. Our conclusion that the genetic risk for type 2 diabetes probably does not differ between the two ethnicities is greatly strengthened by this recent publication.

Figure 3 depicts overlap between genes associated with type 2 diabetes in South Asians and white Europeans. Considerably more signals have been identified in white Europeans due to the greater number of GWAS. Because a majority of signals were replicated in our meta-analysis, it is unlikely that they are unique to white Europeans. Rather, larger GWAS with greater than 36,000 case/control pairs are required to detect ORs as low as 1.05 with an RAF of $10 \%$. The four GWAS in South Asians represent about 25,704 cases and 43,688 controls, therefore more GWAS in South Asians alone will detect small effects and discover SNPs unique to this group, and ultimately facilitate further elucidation of the genetic basis of type 2 diabetes in this group.

We did not find a trend in RAFs; specifically, RAFs were not consistently higher in South Asians. Risk alleles from six SNPs in this analysis were the major/common alleles in both ethnicities. Negative selection usually prevents risk alleles from becoming common unless they are advantageous [50]. In the case of PPARG rs1801282, the risk allele is responsible for increased fat storage and may have been advantageous in ancient environments with unpredictable food supply and high level of activity, but now predisposes to type 2 diabetes [50]. However, to truly establish whether active selection of an allele exists, systematic study of population differentiation is warranted [51].

Testing novel SNPs discovered from South Asians GWAS in white Europeans

Finally, we tested novel SNPs derived from South Asians in white Europeans; only three were associated with type 2 diabetes. Interestingly, one of the novel SNPs, rs1048886, is a functional missense mutation resulting in a change from glutamine to arginine in the UPF0369 protein involved in immune response [52]. Additionally, the G risk allele for $S G C G$ rs9552911 is not present in white Europeans. The putative existence of private polymorphisms in South Asians is supported by presence of assortative mating resulting from prolonged geographical and cultural isolation of this region from white Europeans [53]. Non-association of the remaining seven dimorphic SNPs with type 2 diabetes in white Europeans is quite puzzling as the risk alleles are present in this group. DIAGRAM's large sample ensures reasonable power; for the lowest RAF of all eight SNPs, the DIAGRAM sample had greater than $80 \%$ power to detect an OR of at least 1.07 , and therefore non-association may be due to substantially different LD structures or lower effect sizes in white Europeans because of ethnic specific gene-environment or genegene interactions. We compared the LD structure for the seven SNPs using HapMap data, and while not substantial, $r^{2}$ values with neighbouring SNPs have some differences (for example, $r^{2}$ of rs 1048886 with neighbouring rs 9455158 is 0.75 in white Europeans and 0.87 in South Asians; $r^{2}$ of rs3923113 with neighbouring rs13432797 is 0.93 in white Europeans and 0.64 in South Asians). Fine mapping analyses will determine whether the same functional variants are responsible for increased risk in both groups and if the effect is comparable, or if 


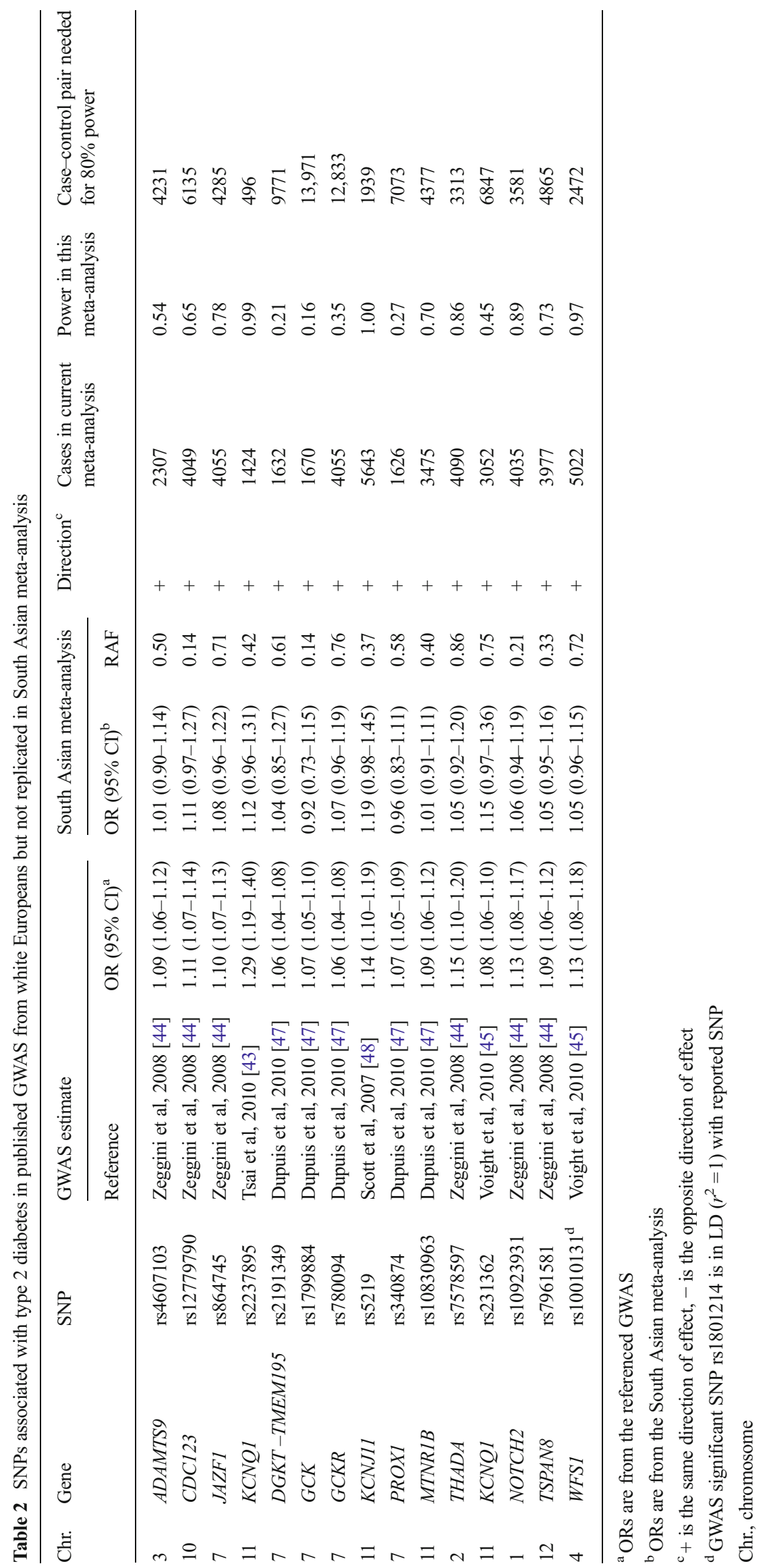


the seven SNPs tag unique functional variants in South Asians.

\section{Population burden}

No difference in genotype score between South Asians and white Europeans was observed, which is consistent with our finding that no trend exists for variation in RAFs. It should be noted that our conclusion is based on an assumption of homogeneity in effect sizes between the groups, supported by our primary analysis. Our conclusion is not consistent with some literature, which shows a greater population burden in South Asians based on unweighted genotype scores [54, 55]. However, while the cumulative genotype score for South Asians was statistically higher than white Europeans in the referenced study, the magnitude of the difference is small ( 0.99 points on a scale that varied from 0 to 32) [54]. Moreover, unweighted scores consider the contribution from SNPs with larger effects to be the same as that from SNPs with small effects, and appear to discriminate less effectively between disease states [56-59]. It should be noted, though, that the genotype score calculation in the referenced paper is much more direct than ours as it is based on primary data.

\section{Strengths and limitations}

This is the first meta-analysis that compares genetic risk of type 2 diabetes in South Asians and white Europeans. Potential limitations of our study include use of unadjusted allelic OR, which precluded us from accounting for SNP-type 2 diabetes associations altered by adiposity. Use of unadjusted allelic OR depended largely on the unavailability of appropriately adjusted data. However, we informally compared adjusted estimates reported in the study with our unadjusted allelic OR and found the adjustment to make little difference; for example, the OR for GCKR rs780094 in the primary report [55] was 0.87 (95\% CI 0.72-1.05) after adjustment for age and sex. In our study, the OR is 0.86 (95\% CI $0.65-1.13)$.

Second, publication and time-lag biases may exist whereby significant results are published more often than negative studies. To minimise these biases, we conducted a thorough search of literature and consulted experts to identify as many eligible studies as possible. We were unable to produce funnel plots to formally assess publication bias because fewer than ten studies were used to meta-analyse a majority of SNPs.

Third, some SNPs in this meta-analysis were only investigated in two cohorts and their summary estimates should be interpreted with caution.

Finally, sample sizes varied with each SNP in South Asians but were consistent for all SNPs in white Europeans, which may create artificial differences between the groups as a result of differences in precision. Our conclusions regarding the genetic heterogeneity between South Asians and white
Europeans are applicable only to genetic risk from bi-allelic common SNPs as our investigation was limited to this type of variant.

\section{Future directions}

The question of why South Asians have a greater risk for type 2 diabetes remains unanswered from a genetic perspective as risk from common SNPs predisposing to type 2 diabetes does not differ. Our results are particularly important as they emphasise the need for future research to explore heritable epigenetic changes, epistasis and gene-environment interactions to answer this question $[60,61]$, rather than focusing on exploring differences in risk estimates and RAFs. Additionally, differences in genetic risk may result from low-frequency causal variants with large effects (rare variants) [62, 63]. The existence of such rare variants can be investigated through candidate gene, whole-genome or whole-exome sequencing. In fact, discovery of rare variants for Crohn's disease greatly supports this paradigm [64]. Large-scale exon resequencing around an MTNRIB SNP modestly associated with type 2 diabetes (OR between 1.10 and 1.15) identified four rare variants that led to loss of melatonin binding and signalling capacity (OR 5.67, 95\% CI 2.17-14.82) [65]. Using a familybased approach with multiple affected members or studying founder populations can facilitate the identification of rare variants in sequencing studies. Finally, systematic reviews and primary studies comparing dietary intake and physical activity patterns between South Asians and white Europeans have noted considerable variation $[66,67]$, which may also contribute to the differences observed between the two ethnic groups.

\section{Conclusions}

Similar effect sizes for SNPs predisposing to type 2 diabetes are apparent among South Asians and white Europeans, but there is variation in RAFs. Additionally, some novel SNPs are present in South Asians. Given the current literature, there is no strong evidence to indicate that currently known genetic variants explain the higher risk of type 2 diabetes in South Asians compared with white Europeans.

Acknowledgements We would like to acknowledge the DIAGRAM consortium investigators for access to genome-wide estimates of SNPs tested for association with type 2 diabetes in white Europeans. We also acknowledge J. Beyene (Department of Mathematics and Statistics, McMaster University, Hamilton, ON, Canada), G. Pond (Department of Oncology, McMaster University), P. G. Joseph (Department of Medicine, McMaster University) and R. J. de Souza (Department of Clinical Epidemiology and Biostatistics, McMaster University) for statistical and technical support. Last, we thank S. Rees (College of Medical and Dental Sciences, University of Birmingham, Birmingham, UK), 
A. Kelly (College of Medical and Dental Sciences, University of Birmingham), D. Sanghera (Department of Pediatrics, University of Oklahoma, Norman, OK, USA), S. Sharma (School of Biotechnology, Shri Mata Vaishno Devi University, Jammu and Kashmir, India), X. Sim (Center for Statistical Genetics, University of Michigan, Ann Arbor, MI, USA) and T. E. Shyong (Department of Medicine, National University of Singapore, Singapore, Republic of Singapore) for providing data required for our meta-analysis.

Funding ZNS is supported by the Ontario Graduate Scholarship and the Canadian Diabetes Association Doctoral Award. SSA holds the Heart and Stroke Foundation of Ontario Michael G. DeGroote endowed Chair in Population Health and a Canada Research Chair in Ethnicity and Cardiovascular Disease. HCG holds the Population Health Research Institute Chair in Diabetes Research sponsored by Aventis, GP holds a Canada Research Chair in Molecular Epidemiology and Genetics, and DM holds a Canada Research Chair in Genetics of Epidemiology.

Duality of interest The authors declare that there is no duality of interest associated with this manuscript.

Contribution statement ZNS made substantial contributions to the design of the study, acquisition of data, conducting the analysis, the interpretation of the data and drafting the article, and provided final approval of the version to be published. SSA made substantial contributions to conception and design and analysis and interpretation of data, critically revised the article for important intellectual content and provided final approval of the version to be published. WQD contributed to the acquisition of data, critically reviewed the article for important intellectual content and provided final approval of the version to be published. GP, DM and HCG substantially contributed to conception and design, critically revised the draft for important intellectual content and provided final approval of the version to be published. SSA is the guarantor of this work.

Open Access This article is distributed under the terms of the Creative Commons Attribution License which permits any use, distribution, and reproduction in any medium, provided the original author(s) and the source are credited.

\section{References}

1. Wild S, Roglic G, Green A et al (2004) Global prevalence of diabetes: estimates for the year 2000 and projections for 2030 . Diabetes Care 27:1047-1053

2. International Diabetes Federation (2013) Diabetes Atlas, 6th edn. International Diabetes Federation, Brussels

3. Stumvoll M, Goldstein BJ, van Haeften TW (2005) Type 2 diabetes: principles of pathogenesis and therapy. Lancet 365:1333-1346

4. Razak F, Anand SS, Shannon H et al (2007) Defining obesity cut points in a multiethnic population. Circulation 115:2111-2118

5. McKeigue PM, Shah B, Marmot MG (1991) Relation of central obesity and insulin resistance with high diabetes prevalence and cardiovascular risk in South Asians. Lancet 337:382-386

6. Raji A, Seely EW, Arky RA, Simonson DC (2001) Body fat distribution and insulin resistance in healthy Asian Indians and Caucasians. J Clin Endocrinol Metab 86:5366-5371

7. Radha V, Mohan V (2007) Genetic predisposition to type 2 diabetes among Asian Indians. Indian J Med Res 125:259-274

8. Kanaya AM, Wassel CL, Mathur D et al (2010) Prevalence and correlates of diabetes in South asian indians in the United States: findings from the metabolic syndrome and atherosclerosis in South asians living in america study and the multi-ethnic study of atherosclerosis. Metab Syndr Relat Disord 8:157-164

9. Little J, Higgins JPT, Ioannidis JPA et al (2009) STrengthening the REporting of Genetic Association Studies (STREGA): an extension of the STROBE statement. PLoS Med 6:e22

10. Lewis CM, Knight J (2012) Introduction to genetic association studies. Cold Spring Harb Protoc 2012:297-306

11. Johnson AD, Handsaker RE, Pulit SL et al (2008) SNAP: a webbased tool for identification and annotation of proxy SNPs using HapMap. Bioinformatics 24:2938-2939

12. Huedo-Medina TB, Sánchez-Meca J, Marín-Martínez F, Botella J (2006) Assessing heterogeneity in meta-analysis: Q statistic or I2 index? Psychol Methods 11:193-206

13. Morris AP, Voight BF, Teslovich TM et al (2012) Large-scale association analysis provides insights into the genetic architecture and pathophysiology of type 2 diabetes. Nat Genet 44:981-990

14. Kooner JS, Saleheen D, Sim X et al (2011) Genome-wide association study in individuals of South Asian ancestry identifies six new type 2 diabetes susceptibility loci. Nat Genet 43:984-989

15. Tabassum R, Chauhan G, Dwivedi OP et al (2013) Genome-wide association study for type 2 diabetes in Indians identifies a new susceptibility locus at 2q21. Diabetes 62:977-986

16. Saxena R, Saleheen D, Been LF et al (2013) Genome-wide association study identifies a novel locus contributing to type 2 diabetes susceptibility in Sikhs of Punjabi origin from India. Diabetes 62:1746-1755

17. Sim X, Ong RT-H, Suo C et al (2011) Transferability of type 2 diabetes implicated loci in multi-ethnic cohorts from Southeast Asia. PLoS Genet 7:e1001363

18. Haseeb A, Iliyas M, Chakrabarti S et al (2009) Single-nucleotide polymorphisms in peroxisome proliferator-activated receptor gamma and their association with plasma levels of resistin and the metabolic syndrome in a South Indian population. J Biosci 34:405-414

19. Anuradha S, Radha V, Mohan V (2011) Association of novel variants in the hepatocyte nuclear factor $4 \mathrm{~A}$ gene with maturity onset diabetes of the young and early onset type 2 diabetes. Clin Genet 80:541-549

20. Chandak GR, Janipalli CS, Bhaskar S et al (2007) Common variants in the TCF7L2 gene are strongly associated with type 2 diabetes mellitus in the Indian population. Diabetologia 50:63-67

21. Chidambaram M, Radha V, Mohan V (2010) Replication of recently described type 2 diabetes gene variants in a South Indian population. Metabolism 59:1760-1766

22. Mukhopadhyaya PN, Acharya A, Chavan Y et al (2010) Metagenomic study of single-nucleotide polymorphism within candidate genes associated with type 2 diabetes in an Indian population. Genet Mol Res 9:2060-2068

23. Janipalli CS, Kumar MVK, Vinay DG et al (2012) Analysis of 32 common susceptibility genetic variants and their combined effect in predicting risk of Type 2 diabetes and related traits in Indians. Diabet Med 29:121-127

24. Rees SD, Hydrie MZI, Shera AS et al (2011) Replication of 13 genome-wide association (GWA)-validated risk variants for type 2 diabetes in Pakistani populations. Diabetologia 54:1368-1374

25. Uma Jyothi K, Jayaraj M, Subburaj KS et al (2013) Association of TCF7L2 gene polymorphisms with T2DM in the population of Hyderabad, India. PLoS One 8:e60212

26. Rees SD, Islam M, Hydrie MZI et al (2011) An FTO variant is associated with Type 2 diabetes in South Asian populations after accounting for body mass index and waist circumference. Diabet Med 28:673-680

27. Bodhini D, Radha V, Dhar M et al (2007) The rs12255372(G/T) and rs 7903146(C/T) polymorphisms of the TCF7L2 gene are associated with type 2 diabetes mellitus in Asian Indians. Metabolism 56:1174 1178

28. Raza ST, Abbas S, Ahmed F et al (2012) Association of MTHFR and PPAR $\gamma 2$ gene polymorphisms in relation to type 2 diabetes mellitus cases among north Indian population. Gene 511:375-379 
29. Tan JT, Ng DPK, Nurbaya S et al (2010) Polymorphisms identified through genome-wide association studies and their associations with type 2 diabetes in Chinese, Malays, and Asian-Indians in Singapore. J Clin Endocrinol Metab 95:390-397

30. Humphries SE, Gable D, Cooper JA et al (2006) Common variants in the TCF7L2 gene and predisposition to type 2 diabetes in UK European Whites, Indian Asians and Afro-Caribbean men and women. J Mol Med (Berl) 84:1005-1014

31. Radha V, Vimaleswaran KS, Babu HNS et al (2006) Role of genetic polymorphism peroxisome proliferator-activated receptor-gamma2 Pro12Ala on ethnic susceptibility to diabetes in South-Asian and Caucasian subjects: Evidence for heterogeneity. Diabetes Care 29: 1046-1051

32. Boodram LG, Miyake K, Hayes MG et al (2011) Association of the KCNJ11 variant E23K with type 2 diabetes in Indo-Trinidadians. West Indian Med J 60:604-607

33. Yajnik CS, Janipalli CS, Bhaskar S et al (2009) FTO gene variants are strongly associated with type 2 diabetes in South Asian Indians. Diabetologia 52:247-252

34. Gupta V, Khadgawat R, Ng HKT et al (2010) A validation study of type 2 diabetes-related variants of the TCF7L2, HHEX, KCNJ11, and ADIPOQ genes in one endogamous ethnic group of north India. Ann Hum Genet 74:361-368

35. Chauhan G, Spurgeon CJ, Tabassum R et al (2010) Impact of common variants of PPARG, KCNJ11, TCF7L2, SLC30A8, HHEX, CDKN2A, IGF2BP2, and CDKAL1 on the risk of type 2 diabetes in 5,164 Indians. Diabetes 59:2068-2074

36. Chauhan G, Tabassum R, Mahajan A et al (2011) Common variants of FTO and the risk of obesity and type 2 diabetes in Indians. J Hum Genet 56:720-726

37. Sanghera DK, Ortega L, Han S et al (2008) Impact of nine common type 2 diabetes risk polymorphisms in Asian Indian Sikhs: PPARG2 (Pro12Ala), IGF2BP2, TCF7L2 and FTO variants confer a significant risk. BMC Med Genet 9:59

38. Lehtovirta M, Kaprio J, Forsblom C et al (2000) Insulin sensitivity and insulin secretion in monozygotic and dizygotic twins. Diabetologia 43:285-293

39. Perry JRB, Voight BF, Yengo L et al (2012) Stratifying type 2 diabetes cases by BMI identifies genetic risk variants in LAMA1 and enrichment for risk variants in lean compared to obese cases. PLoS Genet 8:e1002741

40. Qi Q, Li H, Loos RJF et al (2009) Common variants in KCNQ1 are associated with type 2 diabetes and impaired fasting glucose in a Chinese Han population. Hum Mol Genet 18:3508-3515

41. Unoki H, Takahashi A, Kawaguchi Tet al (2008) SNPs in KCNQ1 are associated with susceptibility to type 2 diabetes in East Asian and European populations. Nat Genet 40:1098-1102

42. Kraft P (2008) Curses-winner's and otherwise-in genetic epidemiology. Epidemiology 19:649-651, discussion 657-8

43. Tsai F-J, Yang C-F, Chen C-C et al (2010) A genome-wide association study identifies susceptibility variants for type 2 diabetes in Han Chinese. PLoS Genet 6:e1000847

44. Zeggini E, Scott LJ, Saxena R et al (2008) Meta-analysis of genomewide association data and large-scale replication identifies additional susceptibility loci for type 2 diabetes. Nat Genet 40:638-645

45. Voight BF, Scott LJ, Steinthorsdottir V et al (2010) Twelve type 2 diabetes susceptibility loci identified through large-scale association analysis. Nat Genet 42:579-589

46. Li H, Gan W, Lu L et al (2013) A genome-wide association study identifies GRK5 and RASGRP1 as type 2 diabetes loci in Chinese Hans. Diabetes 62:291-298

47. Dupuis J, Langenberg C, Prokopenko I et al (2010) New genetic loci implicated in fasting glucose homeostasis and their impact on type 2 diabetes risk. Nat Genet 42:105-116
48. Scott LJ, Mohlke KL, Bonnycastle LL et al (2007) A genome-wide association study of type 2 diabetes in Finns detects multiple susceptibility variants. Science 316:1341-1345

49. DIAbetes Genetics Replication And Meta-analysis (DIAGRAM) Consortium, Asian Genetic Epidemiology Network Type 2 Diabetes (AGEN-T2D) Consortium, South Asian Type 2 Diabetes (SAT2D) Consortium et al (2014) Genome-wide trans-ancestry meta-analysis provides insight into the genetic architecture of type 2 diabetes susceptibility. Nat Genet 46: 234-244

50. Di Rienzo A, Hudson RR (2005) An evolutionary framework for common diseases: the ancestral-susceptibility model. Trends Genet 21:596-601

51. Mattei J, Parnell LD, Lai C-Q et al (2009) Disparities in allele frequencies and population differentiation for 101 diseaseassociated single nucleotide polymorphisms between Puerto Ricans and non-Hispanic whites. BMC Genet 10:45

52. Yang IV, Wade CM, Kang HM et al (2009) Identification of novel genes that mediate innate immunity using inbred mice. Genetics 183 : $1535-1544$

53. Moorjani P, Thangaraj K, Patterson N et al (2013) Genetic evidence for recent population mixture in India. Am J Hum Genet 93:422-438

54. Anand SS, Meyre D, Pare G et al (2013) Genetic information and the prediction of incident type 2 diabetes in a high-risk multiethnic population: the EpiDREAM genetic study. Diabetes Care 36:28362842

55. Rees SD, Hydrie MZI, O'Hare JP et al (2011) Effects of 16 genetic variants on fasting glucose and type 2 diabetes in South Asians: ADCY5 and GLIS3 variants may predispose to type 2 diabetes. PLoS One 6:e24710

56. De Haan HG, Bezemer ID, Doggen CJM et al (2012) Multiple SNP testing improves risk prediction of first venous thrombosis. Blood $120: 656-663$

57. Cooke JN, Ng MCY, Palmer ND et al (2012) Genetic risk assessment of type 2 diabetes-associated polymorphisms in African Americans. Diabetes Care 35:287-292

58. Chen H, Poon A, Yeung C et al (2011) A genetic risk score combining ten psoriasis risk loci improves disease prediction. PLoS One 6: e19454

59. Lin X, Song K, Lim N et al (2009) Risk prediction of prevalent diabetes in a Swiss population using a weighted genetic score-the CoLaus Study. Diabetologia 52:600-608

60. Maher B (2008) Personal genomes: the case of the missing heritability. Nature 456:18-21

61. Manolio TA, Collins FS, Cox NJ et al (2009) Finding the missing heritability of complex diseases. Nature 461:747-753

62. Cirulli ET, Goldstein DB (2010) Uncovering the roles of rare variants in common disease through whole-genome sequencing. Nat Rev Genet 11:415-425

63. Wray NR, Purcell SM, Visscher PM (2011) Synthetic associations created by rare variants do not explain most GWAS results. PLoS Biol 9:e1000579

64. Hugot JP, Chamaillard M, Zouali $\mathrm{H}$ et al (2001) Association of NOD2 leucine-rich repeat variants with susceptibility to Crohn's disease. Nature 411:599-603

65. Bonnefond A, Clément N, Fawcett K et al (2012) Rare MTNR1B variants impairing melatonin receptor $1 \mathrm{~B}$ function contribute to type 2 diabetes. Nat Genet 44:297-301

66. Simmons D, Williams R (2007) Dietary practices among Europeans and different South Asian groups in Coventry. Br J Nutr 78:5

67. Babakus WS, Thompson JL (2012) Physical activity among South Asian women: a systematic, mixed-methods review. Int J Behav Nutr Phys Act 9:150 